\title{
Universal aspects of macromolecules in polymer blends, solutions, and supercritical mixtures
}

\author{
Y. B. Melnichenko, ${ }^{1}$ G. D. Wignall, ${ }^{1}$ and D. Schwahn ${ }^{2}$ \\ ${ }^{1}$ Solid State Division, Oak Ridge National Laboratory, Oak Ridge, Tennessee 37831 \\ ${ }^{2}$ Forschungszentrum Jülich GmbH, D-52425 Jülich, Germany
}

(Received 17 December 2001; published 19 June 2002)

\begin{abstract}
We demonstrate that macromolecules in miscible polymer blends may behave as good, $\Theta$, and poor polymeric solvents for each other. We construct a conceptual phase diagram, delineating the range of validity of the random-phase approximation, outside of which polymers contract or expand beyond their unperturbed dimensions, contrary to common assumptions. Remarkably, the correlation length for polymer blends, solutions, and supercritical mixtures collapses onto a master curve, reflecting universal behavior for macromolecules in polymeric and small-molecule $\Theta$ solvents.
\end{abstract}

DOI: 10.1103/PhysRevE.65.061802

PACS number(s): 61.41.+e, 61.12.Ex

Polymer blends are of increasing scientific and commercial importance, and over the past two decades there has been a significant increase in the use of small-angle neutron scattering (SANS) to determine the miscibility of various macromolecules, based on the de Gennes random-phase approximation (RPA) [1-4]:

$$
S_{t}^{-1}(Q)=S_{s, A}^{-1}(Q)+S_{s, B}^{-1}(Q)-2 \chi
$$

$S_{t}(Q)$ is the total scattering structure factor, which contains information on both intramolecular and intermolecular correlations between polymer segments and is related to the correlation length of the concentration fluctuations, $\xi . S_{s, i}(Q)$ is the single-chain structure factor which contains information on the intramolecular correlations, and thus on the dimensions (e.g., the radius of gyration $R_{g, i}$ ) of the polymer components $(i=A, B)$; the scattering vector is given by $Q$ $=4 \pi \lambda^{-1} \sin \theta$, where $2 \theta$ is the scattering angle and $\lambda$ is the neutron wavelength, and $\chi$ is the Flory interaction parameter which accounts for all nonideal contributions to the free energy of mixing. The RPA [Eq. (1)] is based on the assumption that the dimensions of polymer chains remain unchanged on mixing and retain the unperturbed radius of gyration $R_{g}(\Theta)$ (as in a melt or in a polymer solution at the $\Theta$ temperature). However, several theoretical [5-7], computer simulation $[8,9]$, and experimental results [10-12] have suggested that this assumption may not hold universally and that $R_{g}$ may shrink or expand with temperature $(T)$ or concentration $(\phi)$.

The deviation of $R_{g}$ from $R_{g}(\Theta)$ is well documented in polymer solutions, i.e., mixtures of macromolecules with small molecule solvents (liquids or supercritical fluids [13]). In the "good" solvent domain $(T>\Theta)$, polymer coils swell due to excluded volume interactions, and for $T<\Theta$ (in the "poor" solvent domain) they collapse due to dominating attractive forces between monomers. The effect of temperature on the degree of swelling $\alpha=R_{g} / R_{g}(\Theta)$ is maximal in dilute solutions (weakly interacting chains) and diminishes when $\phi \sim \phi^{*}$, where $\phi^{*}$ is the polymer overlap concentration. The pairwise attractive and repulsive interactions compensate at the $\Theta$ temperature where $R_{g}(\Theta)$ is unperturbed by excluded volume effects and is also independent of the critical concen- tration fluctuations. Considering a polymer blend as a special case of a polymer solution with a high-molecular-weight solvent [14], one might expect that the RPA should be strictly valid only for "ideal" or $\Theta$ blends of noninteracting polymers $(\chi=0)$. To our knowledge, Brereton and Vilgis [5] were the first to suggest that the RPA may break down if the quality of the polymeric solvent becomes sufficiently poor or good and calculated conditions for a "pretransition to a $\Theta$-type state" in a polymer blend. They also envisioned deviations of $R_{g}$ from $R_{g}(\Theta)$, though surprisingly, with only a few exceptions $[12,15,16]$, the concept of the $\Theta$ state and its consequences for the structure-property relationships in polymer blends have not yet been generally appreciated by experimentalists. In this paper, we systematically explore the interrelation between $R_{g}$ and $\xi$ in polymer blends using SANS, and we demonstrate the existence of the $\Theta$ lines in the phase diagrams of polymer blends, in addition to binodals and spinodals traditionally discussed in the literature. The occurrence of the $\Theta$ lines is used to delineate the range of validity of the RPA as well as to explore universal aspects of the polymer behavior in polymeric and small molecule $\Theta$ solvents.

The two competing characteristic lengths $\left(\xi\right.$ and $\left.R_{g}\right)$ in nonoverlapping polymer solutions at the $\Theta$ temperature are related via $[13,17]$

$$
\xi(\Theta)=R_{g}(\Theta) / \sqrt{3} .
$$

Here we extend this relation to polymer blends, which possess an additional characteristic length corresponding to the $R_{g}$ of the polymeric solvent. For "ideal" blends $\chi(T=\Theta)$ $=0$, Eq. (1) may be rewritten as

$$
\begin{aligned}
S_{t}(Q)^{-1}= & {\left[\phi_{A} N_{A} v_{A} S_{D}\left(Q^{2} R_{g, A}^{2}\right)\right]^{-1} } \\
& +\left[\phi_{B} N_{B} v_{B} S_{D}\left(Q^{2} R_{g, B}^{2}\right)\right]^{-1},
\end{aligned}
$$

where $S_{t}(Q)$ is well approximated in the limit of small $Q$ by the Ornstein-Zernike function: 


$$
S_{t}(Q)=S(0) /\left(1+Q^{2} \xi^{2}\right)
$$

and $\phi_{i}, N_{i}$, and $v_{i}$ are the volume fraction, degree of polymerization, and monomer volumes of the components $A$ and $B$, respectively. $S_{D}$ is the Debye function for ideal polymer coils:

$$
S_{D}=\left(2 / y^{2}\right)\left(y-1+e^{-y}\right), \quad y=Q^{2} R_{g, i}^{2} .
$$

Expanding the Debye functions in Eq. (3) in the limit $Q \Rightarrow 0$, we may generalize Eq. (2) and extend it to polymer $\Theta$ blends:

$$
\xi_{k}(\Theta)=R_{g, k}(\Theta) / \sqrt{3},
$$

where

$$
R_{g, k}^{2}(\Theta)=(1+k)^{-1}\left[R_{g, A}^{2}(\Theta)+k R_{g, B}^{2}(\Theta)\right] .
$$

In Eq. (7), $k=\left(\phi_{A} N_{A} v_{A}\right) /\left(\phi_{B} N_{B} v_{B}\right)$ is an "asymmetry factor" which accounts for the cumulative asymmetry between the components. We note that in the strong asymmetry limit $(k \Rightarrow 0, k \Rightarrow \infty)$, as well as in the ideal symmetry limit ( $k$ $=1, R_{g, A}=R_{g, B}$ ), Eq. (2) for the $\Theta$ polymer solutions with a single $R_{g}$ is recovered. Equation (6) may be used for determining the $\Theta$ temperatures from the variation of the correlation length $\xi$ versus $\tau=\left|T-T_{C}\right| / T$, where $T_{C}$ is the critical temperature of phase demixing. Using the mean-field scaling law $\xi=\xi_{\mathrm{Mf}}|\tau|^{-(\nu=0.5)}$ [1] as well as the condition $\xi$ $=\left.\xi_{k}(\Theta)\right|_{T=\Theta}$, it may be shown that

$$
\Theta=T_{C}\left\{1 \mp\left[\xi_{\mathrm{mf}} / \xi_{k}(\Theta)\right]^{2}\right\}^{-1},
$$

where "-" and "+" correspond to upper critical solution temperature (UCST) and lower critical solution temperature (LCST) blends, respectively.

The polymer blends studied were mixtures of deuterated $(d)$ and protonated $(h)$ polydimethylsiloxane $(d+h$ PDMS) with polyethylmethylsiloxane (PEMS), $(h+d)$ polymethylmethacrylate (PMMA)-polyvinylphenol (PVPh), $d$-polystyrene (dPS)-polyphenylmethylsiloxane (PPMS), and dPS-polyvinylmethylether (PVME). $h$ PDMS, $d$ PDMS, PEMS, and PPMS were synthesized and characterized as described previously $[13,18]$. dPS, PVME, $d$ PMMA, and $h$ PMMA were obtained from Polymer Source, Canada and $\mathrm{PVPh}$ was obtained from Aldrich. The polydispersity index of all polymers was generally $M_{W} / M_{N} \leqslant 1.07$, except for $\operatorname{PVPh}\left(M_{W} / M_{N} \sim 2.5\right)$.

The coherent cross section, $I(Q)$, from an incompressible mixture of identical labeled (deuterated) and protonated polymers (component $A$ ) in a protonated polymeric solvent (component $B$ ) is given by $[13,19]$

$$
I(Q, x)=K^{*} S_{s, A}(Q)+L^{*} S_{t}(Q) .
$$

The prefactors $K^{*}$ and $L^{*}$ in Eq. (9) are functions of scattering lengths of the deuterated and protonated monomers of component $A$, the protonated polymeric solvent (component $B)$, as well as the mole fraction $(x)$ of deuterated chains of

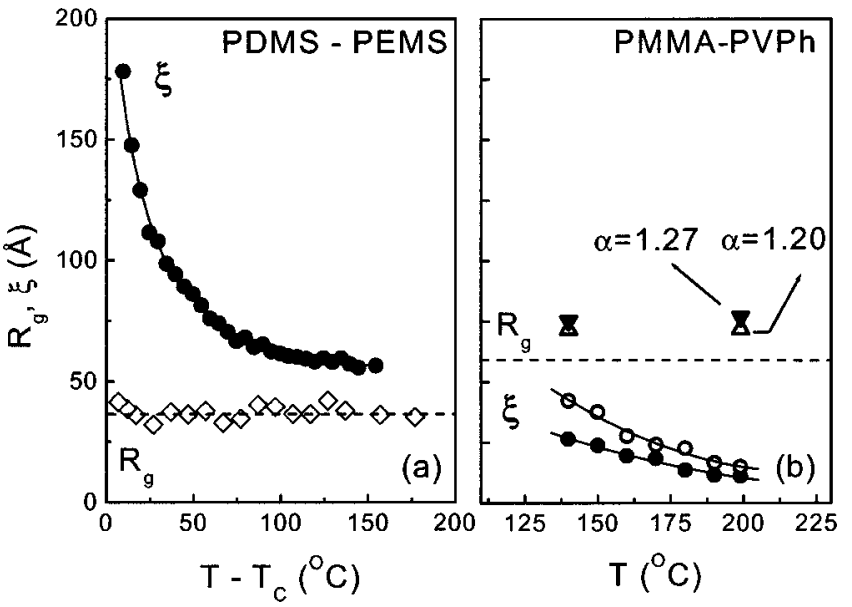

FIG. 1. (a) $R_{g}(\diamond)$ of dPDMS $\left(M_{W}=19500\right)$ in a blend $[0.1$ $d$ PDMS + 0.9 hPDMS $\left.\left(M_{W}=19500\right)\right]$-PEMS $\quad\left(M_{W}=23000\right)$, $\phi_{(h+d) \text { PDMS }}=0.51 ; \xi(\bullet)$ in a blend $d$ PDMS-PEMS, $\phi_{d \text { PDMS }}$ $=0.51$. (b) $R_{g}(\nabla),(\mathbf{\Delta})$ of $d$ PMMA $\left(M_{W}=58500\right)$ in a blend $\left[0.138 d\right.$ PMMA + 0.862 $h$ PMMA $\left.\quad\left(M_{W}=63000\right)\right]-\mathrm{PVPh} \quad\left(M_{W}\right.$ $=8000) ; \xi(\bigcirc),(\bigcirc)$ in a blend $d$ PMMA-PVPh. Open symbols, $\phi_{\text {PMMA }}=0.5$; solid symbols, $\phi_{\text {PMMA }}=0.3$. Dashed lines show $R_{g}(\Theta)_{d \mathrm{PDMS}}=35 \pm 1 \AA$ and $R_{g}(\Theta)_{d \mathrm{PMMA}}=61 \pm 2 \AA$ [20].

component $A$. If all $A$ chains are deuterated $(x=1)$, the prefactor $K^{*}=0$, and one obtains $\xi(T)$ directly from $I(Q, x$ $=1) \sim S_{t}(Q)$. For mixtures of $(d+h)$ PMMA $+\mathrm{PVPh}$, the parameter $L^{*}$, [Eq. (9)] is exactly zero at $x=0.138$ and Eq. (9) gives the single chain scattering directly [20]. For mixtures of $(d+h)$ PDMS and PEMS there is no ratio, $0<x$ $<1$, to achieve "zero averaged contrast" [19] and eliminate $S_{t}(Q, x)$ completely, though its contribution can be minimized by choosing small concentration (e.g., $x \sim 0.1$ ) and subtracting $L^{*} S_{t}(Q)$ [Eq. (9)] as described elsewhere [21].

SANS experiments with PDMS-PEMS blends were carried out at Oak Ridge National Laboratory and the other blends were investigated at the Forschungszentrum, Jülich, Germany. The range of scattering vectors was $0.005<Q$ $<0.03 \AA^{-1}$. The sample temperature was controlled to better than \pm 0.2 and $\pm 0.02 \mathrm{~K}$ in the Oak Ridge and Jülich experiments, respectively. The data were corrected and placed on an absolute scale using procedures described elsewhere [22]. The structure factors $S_{s, A}(Q)$ and $S_{t}(Q)$ were obtained from Eq. (9) and used to calculate $\xi$ and $R_{g}$ at different temperatures using Eqs. (4) and (5).

The temperature variation of $R_{g}$ and $\xi$ in PDMS-PEMS and PMMA-PVPh blends is shown in Fig. 1, where the $R_{g}$ of $d$ PDMS remains constant at all temperatures and the average radii, $R_{g}=37 \pm 3 \AA$, agree with the unperturbed dimensions for $d$ PDMS $R_{g}(\Theta)=35 \AA$ [20]. In contrast, the correlation length varies strongly with temperature, diverging as $T \Rightarrow T_{C} \approx 418.15 \mathrm{~K}\left(\xi \gg R_{g}\right)$, and leveling off $\left(\xi / R_{g} \sim 1.65\right.$ \pm 0.05 ) for $T \gg T_{C}$ [Fig. 1(a)]. Quite the opposite effects are observed in PMMA-PVPh blends, where $d$ PMMA coils are swollen beyond the $\Theta$ dimensions and $\xi$ is much smaller than $R_{g}$ [Fig. 1(b)]. The observed variation of $\xi(T) / R_{g}$ in the weakly interacting PDMS-PEMS blend [Fig. 1(a)] is similar 

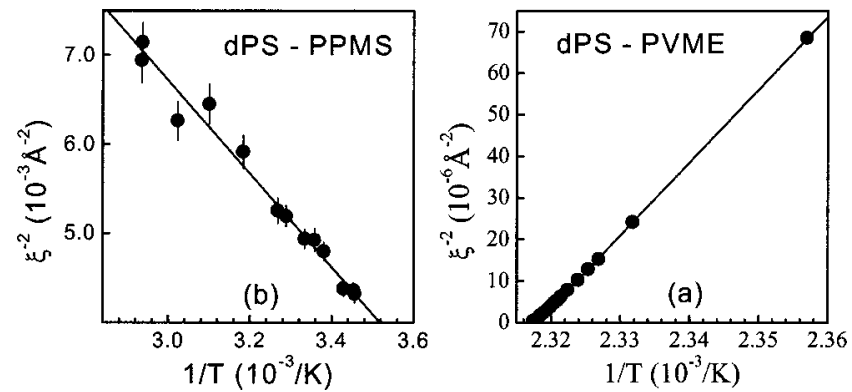

FIG. 2. (a) $\xi^{2}$ vs $T^{1}$ in the LCST blend $d$ PS $\left(M_{W}\right.$ $=246000)-\operatorname{PVME}\left(M_{W}=201000\right), \phi_{d}=0.19$. (b) $\xi^{2}$ vs $T^{1}$ in the UCST blend $d$ PS $\left(M_{W}=2620\right)$-PPMS $\left(M_{W}=2530\right), \phi_{d \mathrm{PS}}=0.48$. Solid lines are described by the mean-field equation $\xi^{-2}=\xi_{\mathrm{mf}}^{-2} \mid 1$ $-T_{C} / T \mid$ with parameters $\xi_{\mathrm{mf}}=15.7 \pm 0.05 \AA, T_{C}=431.15 \pm 0.1 \mathrm{~K}$ (dPS-PVME) $\quad$ and $\quad \xi_{\mathrm{mf}}=6.7 \pm 0.05 \AA, \quad T_{C}=233.75 \pm 0.5 \mathrm{~K}$ ( $d$ PS-PPMS).

to that found in polymer solutions in poor organic solvents (e.g., polystyrene-acetone [17]), which remain microscopically phase-separated $\left(\xi \geqslant R_{g}\right)$ at all temperatures. Likewise, $d$ PDMS and PEMS do not mix randomly and thus PEMS behaves as a poor polymeric solvent for $d$ PDMS. In contrast, the behavior of the hydrogen-bonded blend PMMA-PVPh is similar to that found in polymer solutions in good solvents (e.g., polystyrene-toluene [23]), which are randomly mixed at $T \gg T_{C}$ and thus PVPh is a good polymeric solvent for $d$ PMMA [24].

The variation of $\xi$ with temperature in the LCST blend dPS-PVME $\left(\phi_{\mathrm{dPS}}=0.19\right)$ and the UCST blend dPS-PPMS $\left(\phi_{\mathrm{dPS}}=0.48\right)$ is shown in Fig. 2. Using experimental values $\xi_{\mathrm{mf}}, T_{C}$ [Fig. 2(a)], and $\xi_{k}(\Theta)=81 \AA$ [Eq. (6)], we calculate via Eq. (8) a "lower" $\Theta$ temperature of dPS-PVME $\Theta_{L}$ $\cong 142 \pm 1{ }^{\circ} \mathrm{C}$. $\Theta_{L}$ may also be determined independently via the condition $\chi(\Theta)=0$. Using experimental data [4,25] on $\chi(T)$ for dPS-PVME blends with the same concentration $\phi_{\mathrm{dPS}}=0.19$ and extrapolating to $\chi=0$, we obtain $\Theta_{L}=142$ $\pm 4{ }^{\circ} \mathrm{C}$, which confirms the consistency of both methods for determining the $\Theta_{L}$. Analogous calculations for dPS-PVME blends $[4,25]$ with various $\phi$ and $M_{W}$ of components give the values of $\Theta_{L}$ shown in Fig. 3(a). Similarly, the inset in Fig. 3(b) shows the "upper $\Theta$ temperatures" $\left(\Theta_{U}\right)$ for the UCST blend dPS-PPMS, calculated via Eq. (8) using the parameters $\xi_{\mathrm{mf}}, T_{C}$ [Fig. 2(b)], and $\xi_{k}(\Theta)=7.9 \AA$ [Eq. (6)] obtained in this work and our previous studies $[18,26]$. We note that both $\Theta_{L}$ and $\Theta_{U}$ are independent of the molecular weight of components, but vary with the concentration of dPS due to the perturbation of the segment-segment interactions caused by deuteration [27]. The effect of isotopic substitution is opposite in sign for UCST and LCST blends, respectively, with the latter becoming more miscible (and the former less) as a function of $\phi_{\mathrm{dPS}}$. The magnitude of the effect is also significantly different for entropy-driven (LCST) and enthalpydriven (UCST) phase demixing.

We believe the data presented in Fig. 3(a) are the first demonstration of the existence of the $\Theta_{U}$ and $\Theta_{L}$ lines, which separate poor $(\chi>0)$ and good solvent $(\chi<0)$ domains in the phase diagrams of both LCST and UCST poly- mer blends. As in polymer solutions, in the good polymeric solvent domain $\left(T<\Theta_{L}, T>\Theta_{U}\right)$, excluded volume effects work to expand $R_{g}$ beyond the unperturbed dimensions. This effect is facilitated in compositionally asymmetric blends $\left(k \Rightarrow 0\right.$ or $\left.k \Rightarrow \infty, \phi_{i} \leqslant \phi_{i}^{*}\right)$, where individual chains of the dilute component are subject to minimal screening effects and expand due to a favorable interaction with the good polymeric solvent, as reported in $[11,12]$. Conversely, in the poor polymeric solvent domain $\left(T>\Theta_{L}, T<\Theta_{U}\right)$, the dominating attractive intramolecular interactions between the segments facilitate contraction of the minority component, as was observed in [10]. At the same time, the majority species experience predominantly the environment of the same component and thus exhibit the unperturbed dimensions as in polymer melt at both $T \gg \Theta$ and $T \ll \Theta[6-8,28]$. The deviation of $R_{g}$ from $R_{g}(\Theta)$ in strongly asymmetric blends restricts the applicability of the RPA to the regions shown schematically in the conceptual phase diagram of the weakly interacting UCST and LCST homopolymer blends [Fig. 3(b)], which summarizes the available experimental observations as well as results of computer simulations.

Our results show that polymer blends reproduce main features of the temperature-concentration phase diagram [1] of polymers in small molecule solvents, though the behavior of polymer blends is much richer due the availability of an additional "degree of freedom" related to the molecular weight of the polymeric solvent. Recently, we have demonstrated that the $\Theta$ condition may also be induced in mixtures of polymers with supercritical fluids by varying temperature and/or pressure [13]. Using the de Gennes scaling temperature variable [1] $\tau^{*}=\left(T-T_{C}\right) /\left(\Theta-T_{C}\right)$, which accounts for the temperature distance from both the $\Theta$ temperature and $T_{C}$, we represent the correlation length of the concentration fluctuations in the scaling form

$$
\xi=\xi_{k}(\Theta)\left(\tau^{*}\right)^{\nu^{*}}
$$

with the exponent $\nu^{*}=0.5$ in the mean-field domain $\left(T \Rightarrow \Theta, \tau^{*} \Rightarrow 1\right)$ and $\nu^{*}=0.63$ in the critical region ( $\left.T \Rightarrow T_{C}, \tau^{*} \Rightarrow 1\right)$, and $\xi_{k}(\Theta)$ defined by Eq. (6). As is seen in Fig. 4, the correlation lengths $\xi / \xi_{k}(\Theta)$ of various $\Theta$ systems - both LCST and UCST polymer blends, polymer solutions, and mixtures of polymers with supercritical fluids - collapse on a master curve in the wide range of the molecular weight 2500-200000, $\Theta$ temperature $65-484^{\circ} \mathrm{C}$, and the critical temperature $-40-160^{\circ} \mathrm{C}$. We believe this to be the first demonstration of the universality of the structure and thermodynamic properties of polymer molecules in the $\Theta$ polymeric, liquid, and supercritical solvents.

In summary, we have demonstrated that, like small molecule solvents, polymers may behave as poor, $\Theta$, and good polymeric solvents for the other component of polymer blends, and we suggested a conceptual phase diagram, which outlines the range of validity of the RPA. We have also demonstrated that macromolecules behave universally in polymeric, liquid, and supercritical $\Theta$ solvents over a wide range of thermodynamic parameters. We believe that the occur- 

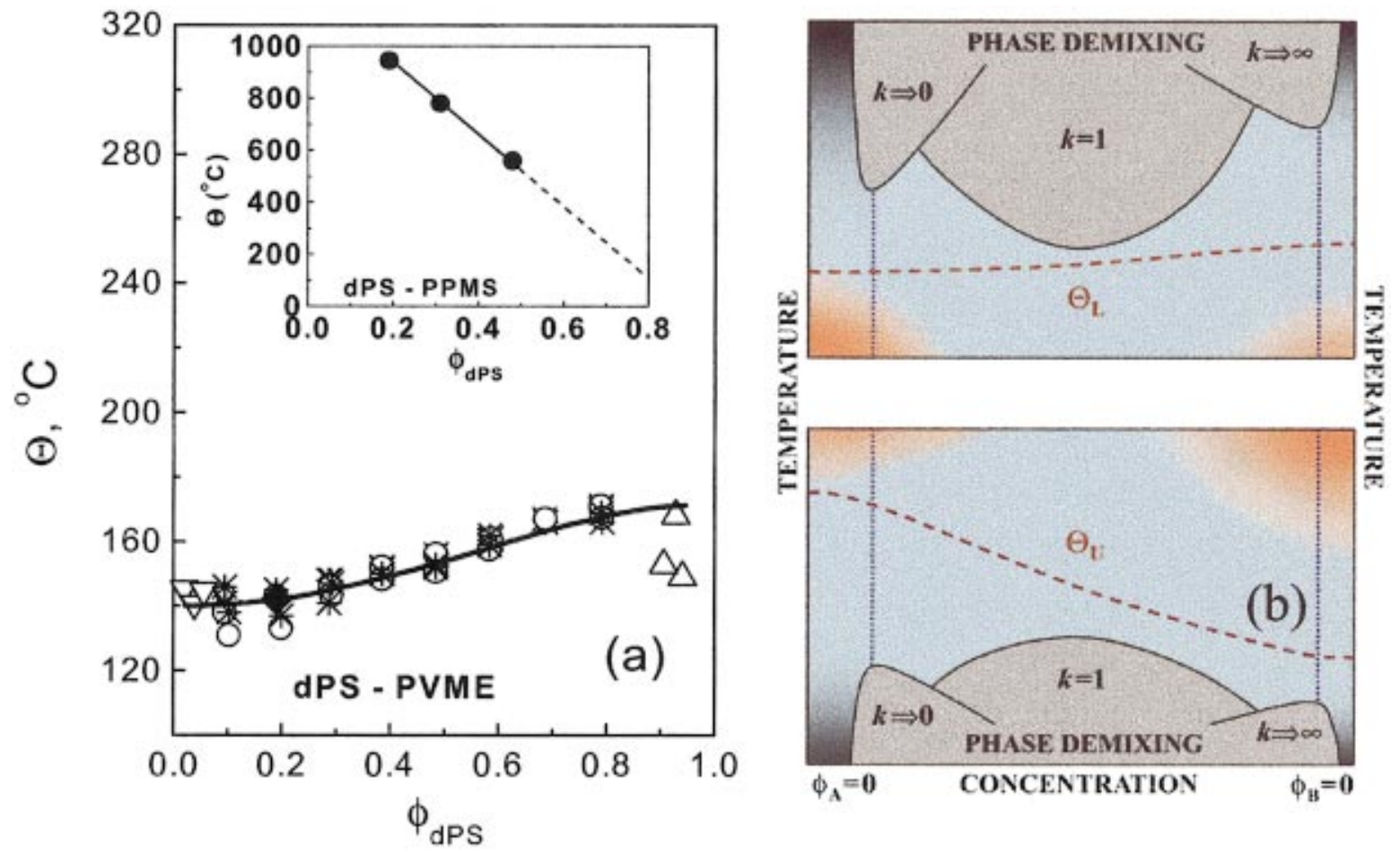

FIG. 3. (Color) (a) Lower $\Theta$ temperatures $\left(\Theta_{L}\right)$ of the LCST blends $d$ PS-PVME obtained using condition $\chi=0(*)$ and Eq. (8) (O) from the data $\chi(T)$ and $\xi(T)$ tabulated in $[4,25] ; \nabla$ and $\triangle$ are $\Theta_{L}$ reported in $[12,16]$. The inset shows the upper $\Theta$ temperatures $\left(\Theta_{U}\right)$ for the UCST blends $d$ PS-PPMS calculated from Eq. (8). Solid lines are guides to the eye. (b) Generic phase diagram of the weakly interacting UCST and LCST $\Theta$ homopolymer blends (one component deuterated). The vertical dotted lines correspond to $\phi=\phi^{*}$ of the dilute component. The RPA is valid in blue domains and breaks down in the strong asymmetry limit (dark blue and reddish domains) due to the shrinking or swelling of polymer coils of the minority component.

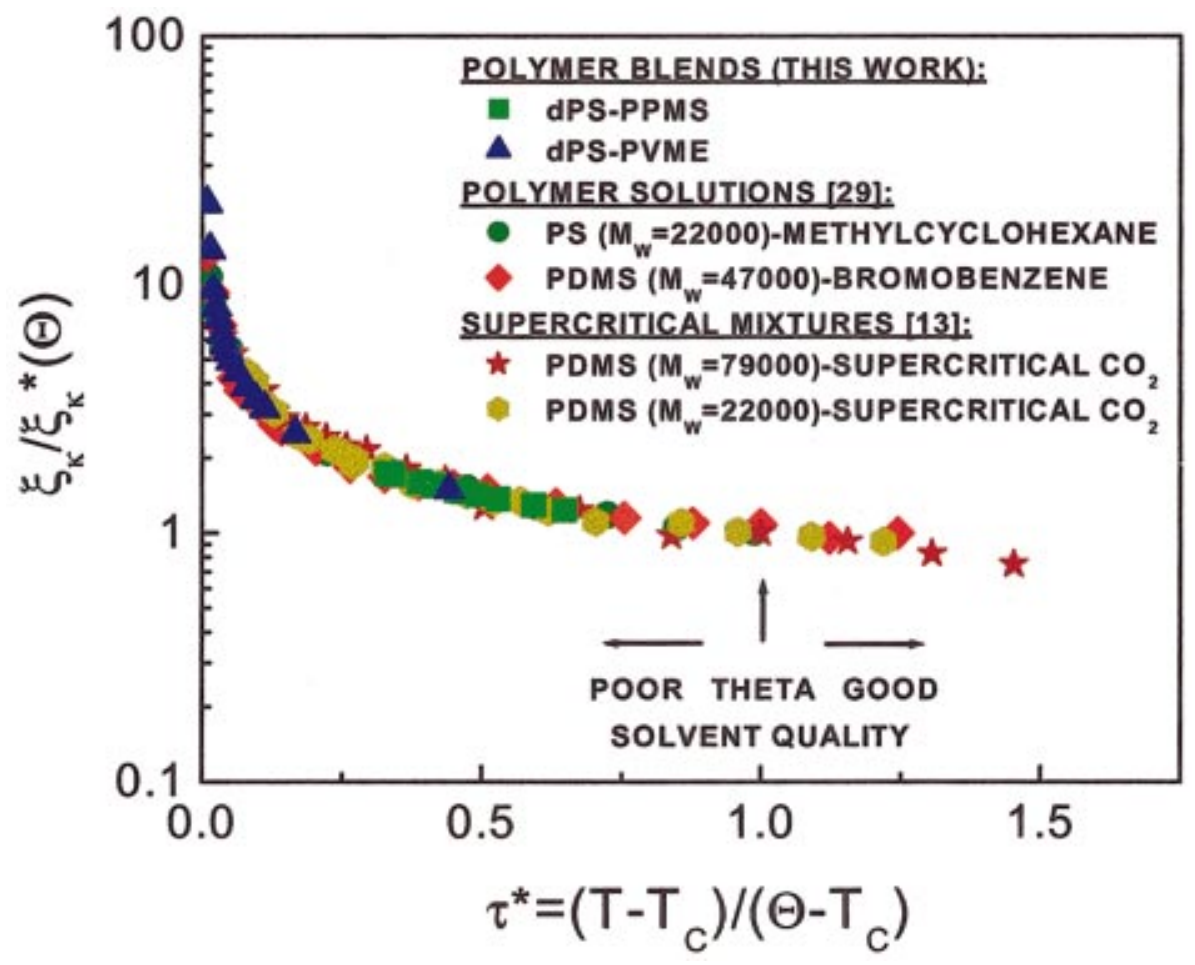

FIG. 4. (Color) Master curve for the correlation length in various systems, which exhibit the $\Theta$ temperatures in their phase diagrams. 
rence of the lower and upper $\Theta$ points as well as their significant variation with the concentration of a deuterated component may provide new insight and interpretation of some of the puzzling phenomena, such as variation of the interaction parameter $\chi$ with $M_{W}$ and composition. The $\Theta$ state concept in polymer blends may also be constructive for rationalizing other important issues, e.g., the $M_{W}$ dependence of the Ginzburg number in polymer blends and the way they may cross over from the mean field to fluctuation regimes. We hope that this work will stimulate further experimental and theoretical efforts, in order to quantify universal and specific aspects of the polymer behavior in the regions of the phase diagram corresponding to the poor, $\Theta$, and good polymeric solvents [29].

This work was supported by the Division of Materials Sciences under Contract No. DE-AC05-00OR22725 with the Oak Ridge National Laboratory, managed by UT-Battelle, LLC. We thank R. M. Briber, J. F. Douglas M. Muthukumar, T. P. Russell, and M. Shibayama for useful discussions and M. Stamm for discussions and providing the isotopically substituted polymers. Experiments of Y.B.M. in Jülich were supported by the Alexander von Humboldt-Stiftung, Germany.
[1] P.-G. De Gennes, Scaling Concepts in Polymer Physics, 2nd ed. (Cornell University Press, Ithaca, 1979).

[2] S. K. Sinha, D. J. Lohse, and M. Y. Lin, Physica B 213, 1 (1995).

[3] D. J. Lohse, R. T. Garner, W. W. Graessley, and R. Krishnamoorti, Rubber Chem. Technol. 72, 569 (1999).

[4] C. C. Han et al., Polymer 29, 2002 (1988).

[5] M. G. Brereton and T. A. Vilgis, J. Phys. (France) 50, 245 (1989).

[6] E. A. Di Marzio and R. M. Briber, Macromolecules 28, 4020 (1995).

[7] G. E. Garas and M. K. Kosmas, J. Chem. Phys. 105, 4789 (1996).

[8] P. Cifra, F. E. Karaz, and W. J. MacKnight, Macromolecules 25, 192 (1992).

[9] K. Binder, Adv. Polym. Sci. 112, 183 (1994).

[10] K. A. Peterson, A. D. Stein, and M. D. Fayer, Macromolecules 23, 111 (1990).

[11] J. M. Lefebvre, R. S. Porter, and G. D. Wignall, Polym. Eng. Sci. 27, 433 (1987).

[12] R. M. Briber, B. J. Bauer, and B. Hammouda, J. Chem. Phys. 101, 2592 (1994).

[13] Y. B. Melnichenko et al., Macromolecules 32, 5344 (1999).

[14] P. J. Flory, J. Chem. Phys. 17, 303 (1949).

[15] D. Schwahn et al., J. Chem. Phys. 87, 6078 (1987).

[16] S. Choi, X. Liu, and R. M. Briber, J. Polym. Sci. [B] 36, 1 (1998).

[17] Y. B. Melnichenko et al., Macromolecules 31, 8436 (1998).

[18] D. Schwahn, K. Mortensen, and S. Jassen, Phys. Rev. Lett. 73, 1452 (1994).

[19] M. Benmouna and B. Hammouda, Prog. Polym. Sci. 22, 49 (1997).
[20] Similarly, unperturbed dimensions of dPDMS $\left[R_{g}(\Theta)\right.$ $=35 \AA]$ and dPMMA $\left[R_{g}(\Theta)=61 \AA\right]$ were measured in the $\Theta$ solvents bromobenzene and acetonitrile, respectively, in separate experiments at the concentration of the labeled chains $(x)$, which satisfies the zero average contrast condition $L^{*}$ $=0$ in each solution.

[21] Y. B. Melnichenko and G. D. Wignall, Phys. Rev. Lett. 78, 686 (1997).

[22] G. D. Wignall and F. S. Bates, J. Appl. Crystallogr. 20, 28 (1987).

[23] J. S. King, W. Boyer, G. D. Wignall, and R. Ullman, Macromolecules 18, 709 (1985).

[24] Strong interactions, such as hydrogen bonding, are known to facilitate miscibility of polymers [M. M. Coleman and P. C. Painter, Prog. Polym. Sci. 20, 1 (1995)]. Our data demonstrate for the first time that the screening effects may be overruled by such interactions and that the PMMA chains in hydrogenbonded blends PMMA-PVPh are swollen even in the concentrated regime.

[25] M. Shibayama, H. Yang, R. S. Stein, and C. C. Han, Macromolecules 18, 2179 (1985).

[26] D. Schwahn and K. Mortensen, in Scattering in Polymer Blends Studied with Small Angle Neutron Scattering, edited by W. Brown and K. Mortensen (Gordon \& Breach, New York, 2000), Chap. 8.

[27] H. Yang, G. Hadziioannou, and R. S. Stein, J. Polym. Sci. [B] 21, 159 (1996).

[28] H. Ito, T. P. Russell, and G. D. Wignall, Macromolecules 20, 2213 (1987).

[29] Y. B. Melnichenko, G. D. Wignall, and W. A. Van Hook, Europhys. Lett. 8, 372 (1999). 